\title{
Prospective Immunophenotyping of CD8+ T-cells and Associated Clinical Outcomes of Patients with Oligometastatic Prostate Cancer Treated with Metastasis-Directed SBRT
}

\author{
Jaden D. Evans, MD¹, Lindsay K. Morris, MD¹, Henan Zhang, MD² $^{2}$, Siyu Cao, MBBS ${ }^{2}$, Xin \\ Liu $^{2}$, Kristin C. Mara, MS ${ }^{3}$, Bradley J. Stish, MD¹, Brian J. Davis, MD PhD ${ }^{1}$, Aaron S. \\ Mansfield, MD ${ }^{4}$, Roxana S. Dronca, MD ${ }^{4,5}$, Matthew J. lott, CNP ${ }^{1}$, Eugene D. Kwon, MD ${ }^{6}$, \\ Robert L. Foote, MD1, Kenneth R. Olivier, MD¹, Haidong Dong, MD PhD\#2,6, and Sean S. \\ Park, MD PhD. ${ }^{\# 1}$ \\ ${ }^{1}$ Department of Radiation Oncology, Mayo Clinic, Rochester, MN \\ ${ }^{2}$ Department of Immunology, Mayo Clinic, Rochester, MN \\ ${ }^{3}$ Division of Biomedical Statistics and Informatics, Mayo Clinic, Rochester, MN \\ ${ }^{4}$ Division of Medical Oncology, Mayo Clinic, Rochester, MN \\ ${ }^{5}$ Division of Medical Oncology, Mayo Clinic, Jacksonville, FL \\ ${ }^{6}$ Department of Urology, Mayo Clinic, Rochester, MN \\ \# These authors contributed equally to this work.
}

\begin{abstract}
Purpose: To study the effects of metastasis-directed stereotactic body radiation therapy (mdSBRT) on $\mathrm{CD}^{+}{ }^{+} \mathrm{T}$-cell subpopulations and to correlate post-mdSBRT immunophenotypic responses with clinical outcomes in patients with oligometastatic prostate cancer (OPCa).

Methods and Materials: Peripheral blood mononuclear cells (PBMCs) were prospectively isolated from 37 patients with OPCa ( $\$$ metastases) treated with mdSBRT. Immunophenotyping identified circulating $\mathrm{CD} 8^{+} \mathrm{T}$-cell subpopulations including: Tumor-Reactive $\left(\mathrm{T}_{\mathrm{TR}}\right)$, Effector Memory $\left(\mathrm{T}_{\mathrm{EM}}\right)$, Central Memory $\left(\mathrm{T}_{\mathrm{CM}}\right)$, Effector $\left(\mathrm{T}_{\mathrm{EF}}\right)$, and Naïve $\left(\mathrm{T}_{\mathrm{N}}\right) \mathrm{T}$-cells from samples collected before and after mdSBRT. Univariate Cox proportional hazards regression was used to assess whether changes in these T-cell subpopulations were potential risk factors for death and/or progression. Kaplan-Meier method was used for survival. Cumulative incidence for progression and new distant metastasis was estimated considering death as a competing risk.
\end{abstract}

Corresponding Author: Sean S. Park, MD PhD, 200 First Street SW, Rochester, MN 55905, Phone: 507-284-4561, Fax: 507-284-0079.

Conflicts of interest: The authors declare no potential conflicts of interest.

Trial registration: This prospective study is registered at ClinicalTrials.gov, number NCT01777802.

Publisher's Disclaimer: This is a PDF file of an unedited manuscript that has been accepted for publication. As a service to our customers we are providing this early version of the manuscript. The manuscript will undergo copyediting, typesetting, and review of the resulting proof before it is published in its final citable form. Please note that during the production process errors may be discovered which could affect the content, and all legal disclaimers that apply to the journal pertain. 
Results: Median follow-up was 39 months (IQR 34-43). Overall survival at 3 years was $78.2 \%$. Cumulative incidence for local progression and new distant metastasis at 3 years was $16.5 \%$ and $67.6 \%$, respectively. Between baseline and day 14 after mdSBRT, an increase in the $\mathrm{T}_{\mathrm{CM}}$ cell subpopulation was associated with the risk of death (HR 1.22 [95\% CI, 1.02-1.47]; $P=0.033$ ), and an increase in the $\mathrm{T}_{\mathrm{TR}}$ cell subpopulation was protective against the risk of local progression (HR 0.80 [95\%CI, 0.65-0.98]; $P=0.032$ ).

Conclusions: An increase in the $\mathrm{T}_{\mathrm{TR}}$ cell subpopulation was protective against the risk of disease progression while an increase in the $\mathrm{T}_{\mathrm{CM}}$ cell subpopulation was associated with the risk of death in patients with OPCa treated with mdSBRT. Disease control may be further improved by better understanding the $\mathrm{CD} 8^{+} \mathrm{T}$-cell subpopulations, and by enhancing their anti-tumor effect.

\section{SUMMARY}

It is unknown how changes in the Tumor-Reactive T-cell subpopulation $\left(\mathrm{CD} 8{ }^{+} \mathrm{PD}-1^{+} \mathrm{CD} 11 \mathrm{a}^{\text {high }}\right)$ correlate with clinical outcomes after metastasis-directed stereotactic body radiation therapy (mdSBRT) for patients with recurrent oligometastatic prostate cancer (OPCa). In this prospective trial of 37 patients with OPCa treated with mdSBRT, an increase in the Tumor-Reactive T-cell subpopulation after mdSBRT was protective against the risk of disease progression. These data have clinical implications for combining mdSBRT with anti-PD-1 therapy and/or adoptive cell transfer procedures.

\section{Keywords}

Oligometastastatic prostate cancer; SBRT; immunophenotyping; metastasis-directed therapy

\section{INTRODUCTION}

Oligometastatic prostate cancer (OPCa) recurrence after radical prostatectomy (RP) and/or radiotherapy (RT) is the most common relapse pattern observed in the modern era. ${ }^{1-4}$ Metastasis-directed therapy (MDT), either in the form of surgery, radiotherapy, or ablation, has been proposed to improve these patients' clinical outcomes. ${ }^{5-7}$ Metastasis-directed stereotactic body radiation therapy (mdSBRT) has been shown to be well-tolerated and to lengthen androgen deprivation therapy (ADT)-free survival in hormone naïve patients as well as delay changes in systemic therapy in castration-resistant patients. ${ }^{1,8}$ Although MDT shows promise in improving clinical outcomes, subclinical metastases and the emergence of new metastatic sites remains problematic.

Combinatorial strategies using both mdSBRT and systemic agents - particularly immunebased therapies and ADT - are actively being investigated to mitigate this problem of disease escape. Many of the proposed combinations involve engaging the patient's CD8 ${ }^{+}$ cytotoxic T-cells and using mdSBRT to augment this response. ${ }^{9-11}$ However, little is known about how mdSBRT affects the circulating CD8 ${ }^{+}$T-cell immunophenotype and whether such phenotypic changes are associated with clinical outcomes such as overall survival, local progression, or the development of new distant metastasis. To evaluate this, we analyzed immunophenotypic changes in circulating $\mathrm{CD}^{+} \mathrm{T}$-cells before and after mdSBRT in 
patients with recurrent OPCa and correlated these changes with clinical oncologic outcomes in a prospective manner.

\section{METHODS AND MATERIALS}

\section{Study Design and Patients}

The protocol was developed as a single-institution, prospective study approved by the Institutional Review Board at our institution. Written informed consent was obtained from all study participants. Eligible patients included male subjects $\geq 18$ years of age; pathologically confirmed PCa patients who had previously undergone curative intent radical prostatectomy (RP), definitive radiotherapy (RT), or both and have prostate-specific antigen (PSA) biochemical relapse per AUA or Phoenix definitions defined by two consecutive PSA levels $>0.2 \mathrm{ng} / \mathrm{mL}$ after $\mathrm{RP}$ or $>2 \mathrm{ng} / \mathrm{mL}$ above the nadir after RT ${ }^{12,13}$; and three or fewer extracranial metastatic lesions diagnosed by ${ }^{11} \mathrm{C}$ choline positron emission tomographycomputed tomography (CholPET-CT) and/or CT-guided biopsy. Patients were excluded if life expectancy was $<3$ months. Patients identified as having castrate-resistant disease, defined as testosterone level $<50 \mathrm{ng} / \mathrm{mL}$, were allowed to enroll on the study. This prospective trial is registered at ClinicalTrials.gov, number NCT01777802.

\section{Isolation of Peripheral Blood Mononuclear Cells}

Whole blood samples of $30-50 \mathrm{~mL}$ were collected via venipuncture using anticoagulant, spray-coated K2EDTA purple-top tubes (BD Biosciences, San Jose, CA) at four time points: baseline prior to mdSBRT, and post-mdSBRT on days 1, 7, and 14. Peripheral blood mononuclear cells (PBMCs) were isolated from buffy coat preparations by density gradient centrifugation using Lymphoprep ${ }^{\mathrm{TM}}$ (Stemcell Technologies, Vancouver, BC) according to well-established immunologic methods. ${ }^{14,15}$ Time points were chosen to analyze PBMCs during the window of greatest presumed change in phenotypic expression, which was estimated to occur between $1-2$ weeks post-SBRT. ${ }^{16}$

\section{Immunophenotyping of CD8+ T-cells}

Immunophenotyping was performed on isolated PBMCs after cell staining with the appropriate monoclonal antibodies (mAbs) (BioLegend®, San Diego, CA) using fluorescence-activated cell sorting (FACS) flow cytometry to identify subpopulations of circulating $\mathrm{CD} 8^{+} \mathrm{T}$-cells. Antibody information used for analysis can be found in Supplementary Table 1. Cell surface staining with mAbs targeting CD8, CD11a, and PD-1 were used to identify tumor-reactive $\mathrm{T}$-cells $\left(\mathrm{T}_{\mathrm{TR}} ; \mathrm{CD} 8{ }^{+} \mathrm{PD}-1^{+} \mathrm{CD} 11 \mathrm{a}^{\text {high }}\right) .{ }^{17,18}$ Cell surface staining with mAbs targeting CCR7 and CD45RA were used to identify effector memory ( $\mathrm{T}_{\mathrm{EM}}$; $\mathrm{CD} 8^{+} \mathrm{CCR}^{-}{ }^{-} \mathrm{CD} 45 \mathrm{RA}^{-}$), central memory $\left(\mathrm{T}_{\mathrm{CM}} ; \mathrm{CD} 8^{+} \mathrm{CCR} 7^{+} \mathrm{CD}^{-}\right.$5RA ${ }^{-}$), effector $\left(\mathrm{T}_{\mathrm{EF}}\right.$ CD8 $\left.{ }^{+} \mathrm{CCR} 7^{-} \mathrm{CD} 45 \mathrm{RA}^{+}\right)$, and Naïve $\left(\mathrm{T}_{\mathrm{N}} \mathrm{CD} 8^{+} \mathrm{CCR} 7^{+} \mathrm{CD} 45 \mathrm{RA}^{+}\right) \mathrm{T}$-cells, respectively. Samples were initially gated on $\mathrm{CD} 8^{+} \mathrm{T}$-cells, and the purity of isolated $\mathrm{CD} 8^{+} \mathrm{T}$-cells was quantified by the FlowJo ${ }^{\circledR}$ analysis platform (FlowJo LLC, Ashland, OR). Within the CD8 ${ }^{+}$ T-cell population, cells were gated on CCR7 and CD45RA and T-cell subpopulation statistics were calculated using the FlowJo® analysis platform. Representative flow cytometry gating strategies used are shown in Supplementary Figure 1. Isotype antibodies were used as negative controls for all experiments. 


\section{Metastasis-Directed Stereotactic Body Radiation Therapy}

Patients were treated with metastasis-directed stereotactic body radiation therapy (mdSBRT) to all oligometastatic lesions. Patients underwent contemporary volumetric planning after CT-based simulation; 4-dimensional CT (4DCT) was used when tumor motion was estimated to be $\geq 1 \mathrm{~cm}$. Patients were simulated and treated in the BodyFIX ${ }^{\circledR}$ vacuum immobilization system (Elekta, Stockholm, Sweden) using a TrueBeam ${ }^{\circledR}$ linear accelerator (Varian, Palo Alto, CA). Target definition and radiotherapy planning have been described previously. ${ }^{19,20}$ In brief, the gross tumor volume (GTV) was defined as all radiographically visible gross disease. For spine lesions, the clinical target volume (CTV) was designed consistent with international consensus guidelines. ${ }^{21}$ For non-spinal osseous lesions, GTV was expanded by 0.5 to $1.0 \mathrm{~cm}$ to include contiguous high-risk bone to create the CTV. For soft tissue or nodal lesions, CTV equaled GTV. CTV was expanded by 0.2 to $0.5 \mathrm{~cm}$ to the planning target volume (PTV). Dose of mdSBRT was determined by the treating radiation oncologist and personalized according to the patient's surrounding normal tissue dose constraints as recommended by the American Association of Physicists in Medicine Task Group 101 (AAPM TG 101). ${ }^{22}$ Four dose regimens were used including 16 Gy in 1 fraction, 18 Gy in 1 fraction, 24 Gy in 1 fraction, or 30 Gy in 3 daily consecutive fractions. Dose was prescribed so that $90 \%$ of the target volume received $\geq 100 \%$ of the prescription dose (D90\% $[\%] \geq 100 \%)$. Target localization was performed with daily cone-beam CT.

\section{Androgen Deprivation Therapy}

ADT was given concurrent with and adjuvant to mdSBRT in patients with castrate-resistant disease, wherein patients were routinely receiving ADT prior to mdSBRT. ADT was most commonly given as an intramuscular (IM) injection of leuprolide acetate (7.5 mg per month) delivered as 3, 4 or 6 month injections. Other ADT regimens used for castrate-resistant disease included oral abiraterone (1,000 $\mathrm{mg}$ daily) in combination with oral prednisone (5 $\mathrm{mg}$ daily), or oral enzalutamide (160 mg daily). Cytotoxic chemotherapy and/or immunotherapy were not used with mdSBRT.

\section{Statistical Analyses}

The data are reported using summary statistics such as mean ( \pm standard deviation [SD]) or median (interquartile range [IQR]) for continuous variables and count (percentage) for categorical variables. Paired t-tests were used to compare baseline blood samples with blood samples after mdSBRT, including in a propensity score matched pair analysis for: 1) patients' $\mathrm{T}_{\mathrm{TR}}$ cell changes who experienced local progression compared with a matched non-local progression group, and 2) patients' $\mathrm{T}_{\mathrm{CM}}$ cell changes who experienced death compared to a matched cohort. Progression-free survival was used as the matching covariate using nearest neighbor matching to reduce bias from this potential confounding variable. A two-sample t-test assuming unequal variance was used in exploratory post hoc subgroup analyses. The survival rates for the outcome of death were estimated using the Kaplan-Meier method. The cumulative incidence for the time-to-event outcomes of local progression and new distant metastasis were estimated considering death as a competing risk for the outcome. Cox proportional hazards regression was used to assess the association of the changes in subpopulations of T-cells with each time-to-event outcome. Hazard ratios (HR) 
are presented with 95\% confidence intervals (CI). Statistical significance was set at $P \leq 0.05$. All reported $P$ values are unadjusted. To account for multiple comparisons, a Bonferroni type I error level of $0.01(=0.05 / 5)$ could be used. All analyses were conducted using SAS version 9.4 (SAS Institute Inc., Cary, NC) and R version 3.2.3 (R Core Team, R Foundation for Statistical Computing, Vienna, Austria).

\section{RESULTS}

The study included 37 patients between January 2013 and October 2014 with recurrent OPCa. Patient, tumor and treatment characteristics are outlined in Table 1. The median follow-up was 39 months (IQR 34-43). The majority of patients had a single oligometastatic lesion treated $(\mathrm{n}=31,84 \%)$. The most commonly treated sites by mdSBRT were non-spinal osseous lesions $(\mathrm{n}=25,68 \%)$. The most common dose regimen was $18 \mathrm{~Gy}$ in a single fraction ( $\mathrm{n}=20,54 \%)$. Median PSA at oligorecurrence was $4.4 \mathrm{ng} / \mathrm{mL}$ (IQR 1.3-6.2) with a median doubling time of 3 months (IQR 1.9-6.2). Primary tumor treatment consisted of radical prostatectomy $(\mathrm{n}=27,73 \%)$, definitive radiotherapy $(\mathrm{n}=5,13.5 \%)$, and prostatectomy followed by adjuvant radiotherapy $(\mathrm{n}=5,13.5 \%)$. ADT concurrent with and adjuvant to mdSBRT was administered in nonrandomized fashion to $19(51 \%)$ patients versus delayed until additional progression in $18(49 \%)$ patients.

Clinical oncologic outcomes are presented in Figure 1. Estimates of overall survival at 1,2, and 3 years were $97.3 \%$ (95\% CI, 92.2-100), 91.9\% (95\% CI, 83.5-100), and 78.2\% (95\% CI, 64.9-94.3), respectively (Fig 1A). Cumulative incidence for any progression, distant or local, at 1, 2, and 3 years were 59.5\% (95\% CI, 40.1-72.6), 64.9\% (95\% CI, 45.6-77.3), and 73.0\% (95\% CI, 54.1-84.1), respectively (Fig 1B). Cumulative incidence for new distant metastasis (NDM) at 1, 2 and 3 years were $54.1 \%$ (95\% CI, 34.8-67.6), 59.5\% (95\% CI, 40.1-72.6), and 67.6\% (95\% CI, 48.4-79.6), respectively (Fig 1C). Cumulative incidence for local progression at the mdSBRT site at 1, 2, and 3 years were 5.4\% (95\% CI, 0-12.4), $10.8 \%$ (95\% CI, 0.2-20.3), and 16.5\% (95\% CI, 3.5-27.8), respectively (Fig 1D). Curves were further dichotomized by castrate-resistant versus hormone-sensitive status, which revealed worse clinical outcomes for patients with castrate-resistant disease (Fig 1). No significant difference in local progression rates by mdSBRT dose and fractionation were observed $(P=0.96$, Table 2$)$.

Prospective $\mathrm{CD} 8^{+} \mathrm{T}$-cell immunophenotyping was performed on the entire cohort before and after mdSBRT at four distinct time points. Upon evaluating T-cell subpopulations from each patient's circulating $\mathrm{CD} 8^{+} \mathrm{T}$-cell pool; $\mathrm{T}_{\mathrm{TR}}, \mathrm{T}_{\mathrm{EM}}, \mathrm{T}_{\mathrm{CM}}, \mathrm{T}_{\mathrm{EF}}$, and $\mathrm{T}_{\mathrm{N}}$ cell subpopulations did not significantly change between baseline and day 1, 7, or 14 after mdSBRT for the entire study group as a whole (Table 3 ). Results were confirmed with the data normalized to the baseline value for each patient's respective T-cell subpopulation $(P>0.05$ for all subpopulations; data not shown). An exception was for $\mathrm{T}_{\mathrm{EF}}$ cells between baseline and day 14 after mdSBRT wherein the mean percent change in $\mathrm{T}_{\mathrm{EF}}$ dropped from $32.6(\mathrm{SD} \pm 17.82)$ to 27.8 (SD \pm 17.27$)(P=0.039$; Table 3$)$, which did not persist after normalization to the baseline value $(P=0.113)$. 
The associated risk between changes in the percentage of circulating $\mathrm{CD} 8^{+} \mathrm{T}$-cell subpopulations before and after mdSBRT and clinical outcomes was studied (Fig 2A-D). Immunophenotypic changes between baseline and day 14 after mdSBRT revealed that an increase in the $\mathrm{T}_{\mathrm{CM}}$ cell subpopulation was associated with the risk of death (HR, 1.22 [95\% CI, 1.02-1.47], $P=0.033$; Fig 2A), and an increase in the $\mathrm{T}_{\mathrm{TR}}$ cell subpopulation was protective against the risk of local progression (HR, 0.80 [95\% CI, 0.65-0.98], $P=0.032$; Fig 2D). Raw mean percentage changes of $\mathrm{T}_{\mathrm{TR}}$ and $\mathrm{T}_{\mathrm{CM}}$ cell subpopulations as a function of time are presented in Figure 3 for time-to-event outcomes of local progression, death, and any progression - local or distant. The general trend observed is a decrease in $\mathrm{T}_{\mathrm{TR}}$ cells for patients who experienced local progression versus an increase in $\mathrm{T}_{\mathrm{TR}}$ cells for patients with locally controlled mdSBRT sites. Furthermore, an observed increase in the $\mathrm{T}_{\mathrm{CM}}$ cells beyond 7 days was seen in the cohort of patients who experienced death. All raw percentage changes for CD8+ T-cell subpopulations between baseline and day 14 post-mdSBRT for each time-to-event outcome are shown in Supplementary Table 4.

Flow cytometry dot plots of $\mathrm{T}_{\mathrm{TR}}$ cell changes for all patients that experienced local progression have been compared to a propensity score matched pair cohort with progression free survival (PFS) as the matching covariate using nearest neighbor matching (Supplementary Figure 2). Raw flow cytometry outputs for $\mathrm{T}_{\mathrm{TR}}$ cell changes from baseline to day 14 are included in Supplementary Table 2. The total mean percent change in $\mathrm{T}_{\mathrm{TR}}$ cells for the matched pair was $-3.81 \%$ for local progressors versus $9.47 \%$ for non-local progressors $(P=0.076)$. The general trend observed in the dot plots is a decrease in $\mathrm{T}_{\mathrm{TR}}$ subset in the 7 patients with local progression versus an increase in the non-local progressing matched cohort.

Flow cytometry dot plots of $\mathrm{T}_{\mathrm{CM}}$ cell changes for all patients that experienced death have been compared to a propensity score matched pair cohort with PFS as the matching covariate (Supplementary Figure 3). Raw flow cytometry outputs for $\mathrm{T}_{\mathrm{CM}}$ cell changes from baseline to day 14 are included in Supplementary Table 3. The total mean percent change in $\mathrm{T}_{\mathrm{CM}}$ cells for the matched pair was $2.61 \%$ for patients who died versus $-2.23 \%$ for patients still alive $(P=0.071)$. The general trend observed in the dot plots is an increase in the $\mathrm{T}_{\mathrm{CM}}$ subset in the 7 different patient patients who died versus a decrease in the no death matched cohort.

In an exploratory post hoc analysis, clinical outcome associations with the following variables were examined: PSA doubling time (PSA-DT) at oligorecurrence, PSA velocity (PSA-V) at oligorecurrence, castrate-resistant status, timing of ADT relative to mdSBRT, and the number of mdSBRT treated sites.

There were $12(32.4 \%)$ patients in the study group identified as having castrate-resistant disease at the time of study enrollment. Of these patients, 9 (75\%) received ADT concurrent with and adjuvant to mdSBRT. The other $3(25 \%)$ did not receive ADT until additional progression after mdSBRT was observed. For the entire cohort, the practice of receiving ADT concurrent with and adjuvant to mdSBRT was found to be protective against the risk of NDM relative to those patients that did not receive ADT until additional progression (HR, 0.44 [95\%CI, 0.200.96], $P=0.039$ ). Mean progression-free survival was significantly 
improved in patients who had PSA-V $\leq 4 \mathrm{ng} / \mathrm{ml} /$ year (23.6 vs. 12.0 months, $P=0.042$ ), noncastrate-resistant disease ( 21.9 vs. 8.8 months, $P=0.012$ ), and in patients who received ADT concurrent with and adjuvant to mdSBRT (24.1 vs. 10.9 months, $P=0.018$ ). Non-significant improvements in mean progressionfree survival were observed in patients who had PSA-DT $\geq 3.5$ months ( 21.8 vs. 13.2 months, $P=0.090$ ), and in patients who had 1 compared to $\geq 2$ mdSBRT treated site(s) (19.2 vs. 9.5 months, $P=0.081)$.

\section{DISCUSSION}

We report on a prospective series of 37 patients with oligometastatic prostate cancer treated with metastasis-directed SBRT who underwent immunophenotyping of identified circulating $\mathrm{CD} 8^{+} \mathrm{T}$-cell subpopulations including: Tumor-Reactive $\left(\mathrm{T}_{\mathrm{TR}}\right)$, Effector Memory $\left(\mathrm{T}_{\mathrm{EM}}\right)$, Central Memory $\left(\mathrm{T}_{\mathrm{CM}}\right)$, Effector $\left(\mathrm{T}_{\mathrm{EF}}\right)$, and Naïve $\left(\mathrm{T}_{\mathrm{N}}\right) \mathrm{T}$-cells from samples collected before and after radiation. This is the first report, to our knowledge, which demonstrates that an increase in the $\mathrm{T}_{\mathrm{TR}}$ cell subpopulation after mdSBRT is associated with OPCa disease control in a prospective human study. Patients with an increase in $\mathrm{T}_{\mathrm{TR}}$ cells from baseline to day 14 after mdSBRT were less likely to have local progression. We also observed that an increase in the $\mathrm{T}_{\mathrm{CM}}$ cell subpopulation during this same time interval was associated with the risk of death. These novel findings have implications for designing combinatorial strategies with mdSBRT and immune-based technologies such as adoptive cell transfer and checkpoint blockade therapies. These data support testing the hypothesis that by expanding and modulating the $\mathrm{T}_{\mathrm{TR}}$ cell pool - either in vivo or ex vivo - the risk of OPCa disease progression may be further reduced. Additional studies are needed to test and validate this hypothesis.

Previous work has shown that CD11a expression is required for the rejection of tumors by $\mathrm{CD}^{+}{ }^{+}$cells. ${ }^{23} \mathrm{CD}^{+} \mathrm{CD} 11 \mathrm{a}^{\text {high }}$ T-cells accumulate within the tumor microenvironment and represent tumor associated antigen (TAA)-specific and tumor-reactive functional cytotoxic T-cell lymphocytes. ${ }^{17}$ The proliferative capacity of $\mathrm{T}_{\mathrm{TR}}$ cells is essential to mount an optimal in vivo antitumor response. ${ }^{24}$ Furthermore, programmed cell death protein 1 (PD-1) expression has been shown to be elevated on $\mathrm{CD} 8^{+} \mathrm{CD} 11 \mathrm{a}^{\text {high }} \mathrm{T}$-cells compared with $\mathrm{CD} 8{ }^{+} \mathrm{CD} 11 \mathrm{a}^{\text {low }} \mathrm{T}$-cells, ${ }^{17}$ and these $\mathrm{T}_{\mathrm{TR}}$ cells $\left(\mathrm{CD} 8{ }^{+} \mathrm{PD}-1^{+} \mathrm{CD} 11 \mathrm{a}^{\text {high }}\right)$ presumably represent the cellular target for anti-PD-1 therapy. ${ }^{17}$ Preclinical models have demonstrated that the capacity of $\mathrm{T}_{\mathrm{TR}}$ cells to control disease is constrained by the persistent expression of PD-1 along with T-cell exhaustion from chronic antigen exposure within the tumor microenvironment. ${ }^{17,25,26}$ Despite these preclinical observations, we have observed that clinical disease control in a prospective human study was improved when there was an increase in this $\mathrm{T}_{\mathrm{TR}}$ cell subpopulation after mdSBRT.

These observations raise the question: "Does the influence of mdSBRT on $\mathrm{T}_{\mathrm{TR}}$ cells alter their antitumor effect?" Preclinical data coupled with our current clinical experience has provided important insights into this question. SBRT has been shown to enhance tumorspecific function of $\mathrm{CD}^{+} \mathrm{CD} 11 \mathrm{a}^{\text {high }} \mathrm{T}$-cells in PD-1 knockout mice; moreover, in these same experiments PD-1 expression restrained the immune-mediated abscopal effect induced by SBRT, which was mitigated by anti-PD-1 therapy. ${ }^{27}$ Previous prospective data showed that combined checkpoint inhibition and palliative radiotherapy to patients with castrate- 
resistant PCa improved progression-free survival compared to radiotherapy alone. ${ }^{28}$ The mechanisms driving the synergistic effect between radiotherapy and checkpoint inhibition are a topic of active investigation and continue to be elucidated. ${ }^{9,10,29}$ Nevertheless, the present study contributes understanding of how mdSBRT influences CD8 ${ }^{+} \mathrm{T}$-cell immunophenotypic changes and how such changes are associated with clinical outcomes. These $\mathrm{T}_{\mathrm{TR}}$ cells' function and associated disease control could, in theory, be further potentiated by anti-PD-1 therapy in addition to their clonal expansion. Clonal expansion could be explored using adoptive cell transfer procedures wherein the identified $\mathrm{T}_{\mathrm{TR}}$ cell subpopulation would be expanded ex vivo. ${ }^{30,31}$ One of the many challenges facing immunooncology currently deals with the appropriate selection of patients for checkpoint inhibition therapy, and which patient population will derive the greatest benefit. One possible solution would be to improve drug delivery to the cellular target, which in the case of anti-PD-1 therapy would be the $\mathrm{T}_{\mathrm{TR}}$ cell. Therefore, by improving anti-PD-1 drug delivery to $\mathrm{T}_{\mathrm{TR}}$ cells or by expanding the number of $\mathrm{T}_{\mathrm{TR}}$ cells upon which anti-PD-1 therapy may target, the therapeutic benefit could be enhanced.

The significance of the association between an increase in the $\mathrm{T}_{\mathrm{CM}}$ cell subpopulation and the risk of death is uncertain. A concept previously introduced by investigators has suggested that some therapeutic cancer vaccines fail because they amplify the corrupted, as opposed to the beneficial, $\mathrm{CD} 8^{+}$memory T-cell subpopulation formed during states of chronic antigen exposure like the tumor-bearing state. ${ }^{24}$ The observed association between $\mathrm{T}_{\mathrm{CM}}$ and the risk of death may indeed represent the deleterious clinical effects of a corrupted memory T-cell subpopulation being amplified post-mdSBRT, resulting in worse clinical outcomes. This is an area of active investigation with the aim of better understanding this subset of T-cells, which may also represent a T-cell subpopulation that could be targeted to improve clinical outcomes.

Once metastatic prostate cancer has been established, oligometastatic biopsies are not commonly performed in routine clinical oncology. In the modern era, advanced imaging techniques using prostate-specific PET radiotracers (e.g. C-11 Choline, PSMA, Fluciclovine) are beginning to influence treatment decisions without histologic confirmation. ${ }^{35}$ However, this monomodal assessment of disease progression and response is flawed by inherent false positive results without histologic confirmation. Liquid biopsies can be sampled non-invasively from a patient's blood and holds the potential to monitor treatment response and relapse in combination with advanced imaging. Some of the most promising actionable biomarkers for metastatic prostate cancer, apart from PSA, include proteomic/ genomic biomarkers, circulating tumor cells (CTC), cell-free tumor DNA (CtDNA) and immunologic biomarkers. ${ }^{36,37}$ Historically, prostate cancer has not been considered an immunogenic tumor. Recent data suggests that prostate cancer expresses multiple tumorassociated antigens (TAAs) including PSA, PSMA, and prostatic acid phosphatase, which may be more immunogenic than previously thought. ${ }^{38}$ To this point, phase III clinical trials, including the landmark IMPACT trial evaluating Sipuleucel-T, have exploited prostate cancer's immunogenicity into an overall survival benefit for asymptomatic or minimally symptomatic metastatic castrate-resistant prostate cancer (mCRPC) patients. ${ }^{39,40}$ Most of the cellular immune response studies to immunotherapeutic agents have been cancer vaccine trials that use biomarkers based on T-cell response to TAAs. Heiser et al showed that human 
autologous dendritic cells which were genetically engineered to express PSA stimulated prostate-specific cytotoxic T-cells in vitro. ${ }^{41}$ Marshall et al reported a Th-1 skewed response utilizing an in vivo PSA-specific DNA vaccine. ${ }^{42}$ In the Onyvax phase II vaccine trial in mCRPC, patients that responded to re-stimulation with vaccine lysate were found to have a Th- 1 cytokine release profile. ${ }^{43}$ In the PROSTVAC phase II vaccine trial, patients with a 6fold increase in T-cell response trended towards improved overall survival. ${ }^{44}$ Similar to the vaccine trials, we observed an augmented specific T-cell response after the therapeutic intervention which resulted in improved oncologic outcomes. In contrast to these vaccine studies, our study did not evaluate the $\mathrm{CD} 4^{+}$compartment, including Th- 1 or Th-2, but rather focused on the major $\mathrm{CD} 8^{+} \mathrm{T}$-cell subpopulations. Updated technology such as mass cytometry or CyTOF allows provision for multiple probes (i.e. >30) to be employed on a single biologic sample which may allow more comprehensive immunophenotyping. ${ }^{45} \mathrm{~A}$ similar experiment employing deep immune profiling in patients treated with metastasisdirected SBRT using mass cytometry could add important information to our results by allowing simultaneous monitoring of all major $\mathrm{T}$ cell subsets including $\mathrm{T}$ helper $(\mathrm{Th})$ cell subsets (CD4 ${ }^{+}$and $\mathrm{CD}^{+}$naïve, central memory, effector, and effector memory), CD4 ${ }^{+}$ regulatory T cells, B cell subsets (naïve, memory, and transitional), plasmablasts, natural killer (NK) cells, gamma delta (gd) T cells, monocytes, dendritic cells, and granulocytes.

The first randomized trial to compare surveillance against metastasis-directed therapy in patients with OPCa recently reported an improvement in ADT-free survival with metastasisdirected therapy. ${ }^{1}$ Metastasis-directed therapy was comprised predominantly of SBRT $(\mathrm{n}=25)$, but also included metastasectomy $(\mathrm{n}=6)$. The present study compliments the work of Ost et al. by furthering the field's understanding of the immunologic changes observed with mdSBRT. Building on these prospective experiences, phase III trials should be designed to further improve both oncologic outcomes and quality of life in OPCa using mdSBRT.

There are limitations with the current study. First, although the study was prospectively conducted, there was significant heterogeneity in the recurrent OPCa patient population. We allowed inclusion of castrate-resistant disease and did not mandate the use or disuse of ADT. The study population was balanced with approximately half the patients receiving ADT concurrent with and adjuvant to mdSBRT compared to half the patients not receiving ADT until additional disease progression. This treatment variation presented an opportunity for interesting exploratory analyses relating to the recent trial of metastasis-directed therapy versus surveillance in the OPCa space. ${ }^{1}$ However, these analyses are hypothesis-generating only, and were not planned subgroup analyses. Therefore, conclusions regarding these subgroups should be tempered until properly designed prospective studies formally address clinical outcomes and their associations with PSA-DT at oligorecurrence, PSA-V at oligorecurrence, castrate-resistant status, timing of ADT relative to mdSBRT, and the number of mdSBRT treated sites. Furthermore, although we are confident that the correlative lab analysis was not influenced by steroids, enzalutamide, or abiraterone given that none of the ADT-receiving patients received these systemic agents sooner than 3 months post-SBRT, which was after the blood collection period. We recognize that the effects Lupron may have on T-cell phenotype are uncertain, which could have influenced our results since there were approximately half the patients receiving this form of ADT concurrent with and immediately adjuvant to mdSBRT. Second, diagnosis of oligorecurrence using 
CholPET-CT has been shown to have high operational characteristics. However, other prostate-specific PET radiotracers including ${ }^{68} \mathrm{Ga} /{ }^{18} \mathrm{~F}$ prostate-specific membrane antigen (PSMA) and fluciclovine PET-CT may be better suited in certain situations to detect and appropriately target PCa oligorecurrence that may not be as readily identified by CholPET$\mathrm{CT}^{35}$

\section{CONCLUSIONS}

In summary, this prospective human study is the first report to demonstrate that an increase after mdSBRT in the $\mathrm{T}_{\mathrm{TR}}$ cell subpopulation, which is the cellular target of anti-PD-1 therapy, is associated with OPCa disease control. Further improvements in disease control could be gained by better understanding the $\mathrm{CD} 8^{+} \mathrm{T}$-cell subpopulations, and by enhancing their antitumor effect.

\section{Supplementary Material}

Refer to Web version on PubMed Central for supplementary material.

\section{Acknowledgments}

Funding: This work was supported by the National Cancer Institute [NCI Grant R01CA200551]; the National Institute of Health [NIH Grant K12CA090628]; the Richard M. Schulze Family Foundation; and the Mayo Clinic Center for Individualized Medicine Biomarker Discovery (IMPRESS) program.

\section{REFERENCES}

1. Ost P, Reynders D, Decaestecker K, Fonteyne V, Lumen N, De Bruycker A, et al. Surveillance or Metastasis-Directed Therapy for Oligometastatic Prostate Cancer Recurrence: A Prospective, Randomized, Multicenter Phase II Trial. J Clin Oncol. 2018;36(5):446-453. [PubMed: 29240541]

2. Parker WP, Davis BJ, Park SS, Olivier KR, Choo R, Nathan MA, et al. Identification of Site-specific Recurrence Following Primary Radiation Therapy for Prostate Cancer Using C-11 Choline Positron Emission Tomography/Computed Tomography: A Nomogram for Predicting Extrapelvic Disease. Eur Urol. 2017;71(3):340-348. [PubMed: 27597240]

3. Sobol I, Zaid HB, Haloi R, Mynderse LA, Froemming AT, Lowe VJ, et al. Contemporary Mapping of Post-Prostatectomy Prostate Cancer Relapse with 11C-Choline Positron Emission Tomography and Multiparametric Magnetic Resonance Imaging. J Urol. 2017;197(1):129-134. [PubMed: 27449262]

4. Parker WP, Evans JD, Stish BJ, Park SS, Olivier K, Choo R, et al. Patterns of Recurrence After Postprostatectomy Fossa Radiation Therapy Identified by C-11 Choline Positron Emission Tomography/Computed Tomography. Int J Radiat Oncol Biol Phys. 2017;97(3):526-535. [PubMed: 28126302]

5. Corbin KS, Hellman S, Weichselbaum RR. Extracranial oligometastases: a subset of metastases curable with stereotactic radiotherapy. J Clin Oncol. 2013;31(11):1384-1390. [PubMed: 23460715]

6. De Bruycker A, Lambert B, Claeys T, Delrue L, Mbah C, De Meerleer G, et al. Prevalence and prognosis of low-volume, oligorecurrent, hormone-sensitive prostate cancer amenable to lesion ablative therapy. BJU Int. 2017;120(6):815-821. [PubMed: 28646594]

7. Gundem G, Van Loo P, Kremeyer B, Alexandrov LB, Tubio JMC, Papaemmanuil E, et al. The evolutionary history of lethal metastatic prostate cancer. Nature. 2015;520(7547):353-357. [PubMed: 25830880]

8. Ost P, Bossi A, Decaestecker K, De Meerleer G, Giannarini G, Karnes RJ, et al. Metastasis-directed therapy of regional and distant recurrences after curative treatment of prostate cancer: a systematic review of the literature. Eur Urol. 2015;67(5):852-863. [PubMed: 25240974] 
9. Bernstein MB, Krishnan S, Hodge JW, Chang JY. Immunotherapy and stereotactic ablative radiotherapy (ISABR): a curative approach? Nat Rev Clin Oncol. 2016;13(8):516-524. [PubMed: 26951040]

10. Sharabi AB, Lim M, DeWeese TL, Drake CG. Radiation and checkpoint blockade immunotherapy: radiosensitisation and potential mechanisms of synergy. Lancet Oncol. 2015;16(13):e498-509. [PubMed: 26433823]

11. Bernstein MB, Garnett CT, Zhang H, Velcich A, Wattenberg MM, Gameiro SR, et al. Radiationinduced modulation of costimulatory and coinhibitory T-cell signaling molecules on human prostate carcinoma cells promotes productive antitumor immune interactions. Cancer Biother Radiopharm. 2014;29(4):153-161. [PubMed: 24693958]

12. Freedland SJ, Rumble RB, Finelli A, Chen RC, Slovin S, Stein MN, et al. Adjuvant and salvage radiotherapy after prostatectomy: American Society of Clinical Oncology clinical practice guideline endorsement. J Clin Oncol. 2014;32(34):3892-3898. [PubMed: 25366677]

13. Cornford P, Bellmunt J, Bolla M, Briers E, De Santis M, Gross T, et al. EAU-ESTROSIOG Guidelines on Prostate Cancer. Part II: Treatment of Relapsing, Metastatic, and CastrationResistant Prostate Cancer. Eur Urol. 2017;71(4):630-642. [PubMed: 27591931]

14. Harris R, Ukaejiofo EO. Rapid preparation of lymphocytes for tissue-typing. Lancet. 1969;2(7615):327.

15. Willinger T, Freeman T, Hasegawa H, McMichael AJ, Callan MF. Molecular signatures distinguish human central memory from effector memory CD8 T cell subsets. J Immunol. 2005;175(9):58955903. [PubMed: 16237082]

16. Werner LR, Kler JS, Gressett MM, Riegert M, Werner LK, Heinze CM, et al. Transcriptionalmediated effects of radiation on the expression of immune susceptibility markers in melanoma. Radiother Oncol. 2017;124(3):418-426. [PubMed: 28893414]

17. Liu X, Gibbons RM, Harrington SM, Krco CJ, Markovic SN, Kwon ED, et al. Endogenous tumorreactive CD8(+) T cells are differentiated effector cells expressing high levels of CD11a and PD-1 but are unable to control tumor growth. Oncoimmunology. 2013;2(6):e23972. [PubMed: 23894697]

18. Dronca RS, Liu X, Harrington SM, Chen L, Cao S, Kottschade LA, et al. T cell Bim levels reflect responses to anti-PD-1 cancer therapy. JCI Insight. 2016;1(6)(6):e86014. [PubMed: 27182556]

19. Ahmed KA, Stauder MC, Miller RC, Bauer HJ, Rose PS, Olivier KR, et al. Stereotactic body radiation therapy in spinal metastases. Int J Radiat Oncol Biol Phys. 2012;82(5):e803-809. [PubMed: 22330988]

20. Muldermans JL, Romak LB, Kwon ED, Park SS, Olivier KR. Stereotactic Body Radiation Therapy for Oligometastatic Prostate Cancer. Int J Radiat Oncol Biol Phys. 2016;95(2):696-702. [PubMed: 27131082]

21. Cox BW, Spratt DE, Lovelock M, Bilsky MH, Lis E, Ryu S, et al. International Spine Radiosurgery Consortium consensus guidelines for target volume definition in spinal stereotactic radiosurgery. Int J Radiat Oncol Biol Phys. 2012;83(5):e597-605. [PubMed: 22608954]

22. Benedict SH, Yenice KM, Followill D, Galvin JM, Hinson W, Kavanagh B, et al. Stereotactic body radiation therapy: the report of AAPM Task Group 101. Med Phys. 2010;37(8):4078-4101. [PubMed: 20879569]

23. Schmits R, Kundig TM, Baker DM, Shumaker G, Simard JJ, Duncan G, et al. LFA-1deficient mice show normal CTL responses to virus but fail to reject immunogenic tumor. J Exp Med. 1996;183(4):1415-1426. [PubMed: 8666900]

24. Klebanoff CA, Gattinoni L, Restifo NP. CD8(+) T-cell memory in tumor immunology and immunotherapy. Immunological reviews. 2006;211:214-224. [PubMed: 16824130]

25. Baitsch L, Baumgaertner P, Devevre E, Raghav SK, Legat A, Barba L, et al. Exhaustion of tumorspecific CD8(+) T cells in metastases from melanoma patients. J Clin Invest. 2011;121(6):2350 2360. [PubMed: 21555851]

26. Dong H, Strome SE, Salomao DR, Tamura H, Hirano F, Flies DB, et al. Tumorassociated B7-H1 promotes T-cell apoptosis: a potential mechanism of immune evasion. Nat Med. 2002;8(8):793800. [PubMed: 12091876] 
27. Park SS, Dong H, Liu X, Harrington SM, Krco CJ, Grams MP, et al. PD-1 Restrains RadiotherapyInduced Abscopal Effect. Cancer immunology research. 2015;3(6):610619.

28. Kwon ED, Drake CG, Scher HI, Fizazi K, Bossi A, van den Eertwegh AJ, et al. Ipilimumab versus placebo after radiotherapy in patients with metastatic castrationresistant prostate cancer that had progressed after docetaxel chemotherapy (CA184-043): a multicentre, randomised, double-blind, phase 3 trial. Lancet Oncol. 2014;15(7):700712.

29. Mansfield AS, Park SS, Dong H. Synergy of cancer immunotherapy and radiotherapy. Aging (Albany NY). 2015;7(3):144-145. [PubMed: 25868470]

30. Drake CG. Prostate cancer as a model for tumour immunotherapy. Nature reviews. Immunology. 2010;10(8):580-593.

31. Restifo NP, Dudley ME, Rosenberg SA. Adoptive immunotherapy for cancer: harnessing the T cell response. Nat Rev Immunol. 2012;12(4):269-281. [PubMed: 22437939]

32. Dronca RS, Mansfield AS, Liu X, Harrington S, Enninga EA, Kottschade LA, et al. Bim and soluble PD-L1 (sPD-L1) as predictive biomarkers of response to anti-PD-1 therapy in patients with melanoma and lung carcinoma. J Clin Oncol. 2017;35:Suppl; abstr 11534.

33. Mansfield AS, Aubry MC, Moser JC, Harrington SM, Dronca RS, Park SS, et al. Temporal and spatial discordance of programmed cell death-ligand 1 expression and lymphocyte tumor infiltration between paired primary lesions and brain metastases in lung cancer. Annals of Oncology. 2016;27(10):1953-1958. [PubMed: 27502709]

34. Terra S, Mansfield AS, Dong H, Peikert T, Roden AC. Temporal and spatial heterogeneity of programmed cell death 1-Ligand 1 expression in malignant mesothelioma. Oncoimmunology. 2017;6(11):e1356146. [PubMed: 29147606]

35. Evans JD, Jethwa KR, Ost P, Williams S, Kwon ED, Lowe VJ, et al. Prostate cancerspecific PET radiotracers: A review on the clinical utility in recurrent disease. Pract Radiat Oncol. 2018;8(1): 28-39. [PubMed: 29037965]

36. Hegemann M, Stenzl A, Bedke J, Chi KN, Black PC, Todenhofer T. Liquid biopsy: ready to guide therapy in advanced prostate cancer? BJU Int. 2016;118(6):855-863. [PubMed: 27430478]

37. Gaudreau P-O, Stagg J, Soulières D, Saad F. The Present and Future of Biomarkers in Prostate Cancer: Proteomics, Genomics, and Immunology Advancements. Biomarkers in Cancer. 2016;8(Suppl 2):15-33.

38. Fernandez-Garcia EM, Vera-Badillo FE, Perez-Valderrama B, Matos-Pita AS, Duran I. Immunotherapy in prostate cancer: review of the current evidence. Clin Transl Oncol. 2015;17(5): 339-357. [PubMed: 25480118]

39. Small EJ, Schellhammer PF, Higano CS, Redfern CH, Nemunaitis JJ, Valone FH, et al. Placebocontrolled phase III trial of immunologic therapy with sipuleucel-T (APC8015) in patients with metastatic, asymptomatic hormone refractory prostate cancer. J Clin Oncol. 2006;24(19):30893094. [PubMed: 16809734]

40. Kantoff PW, Higano CS, Shore ND, Berger ER, Small EJ, Penson DF, et al. Sipuleucel-T immunotherapy for castration-resistant prostate cancer. N Engl J Med. 2010;363(5):411422.

41. Heiser A, Maurice MA, Yancey DR, Wu NZ, Dahm P, Pruitt SK, et al. Induction of polyclonal prostate cancer-specific CTL using dendritic cells transfected with amplified tumor RNA. J Immunol. 2001;166(5):2953-2960. [PubMed: 11207244]

42. Marshall DJ, San Mateo LR, Rudnick KA, McCarthy SG, Harris MC, McCauley C, et al. Induction of Th1-type immunity and tumor protection with a prostate-specific antigen DNA vaccine. Cancer Immunol Immunother. 2005;54(11):1082-1094. [PubMed: 16047142]

43. Michael A, Ball G, Quatan N, Wushishi F, Russell N, Whelan J, et al. Delayed disease progression after allogeneic cell vaccination in hormone-resistant prostate cancer and correlation with immunologic variables. Clin Cancer Res. 2005;11(12):4469-4478. [PubMed: 15958632]

44. Gulley JL, Arlen PM, Madan RA, Tsang KY, Pazdur MP, Skarupa L, et al. Immunologic and prognostic factors associated with overall survival employing a poxviral-based PSA vaccine in metastatic castrate-resistant prostate cancer. Cancer Immunol Immunother. 2010;59(5):663-674. [PubMed: 19890632]

45. Bendall SC, Nolan GP, Roederer M, Chattopadhyay PK. A deep profiler's guide to cytometry. Trends Immunol. 2012;33(7):323-332. [PubMed: 22476049] 

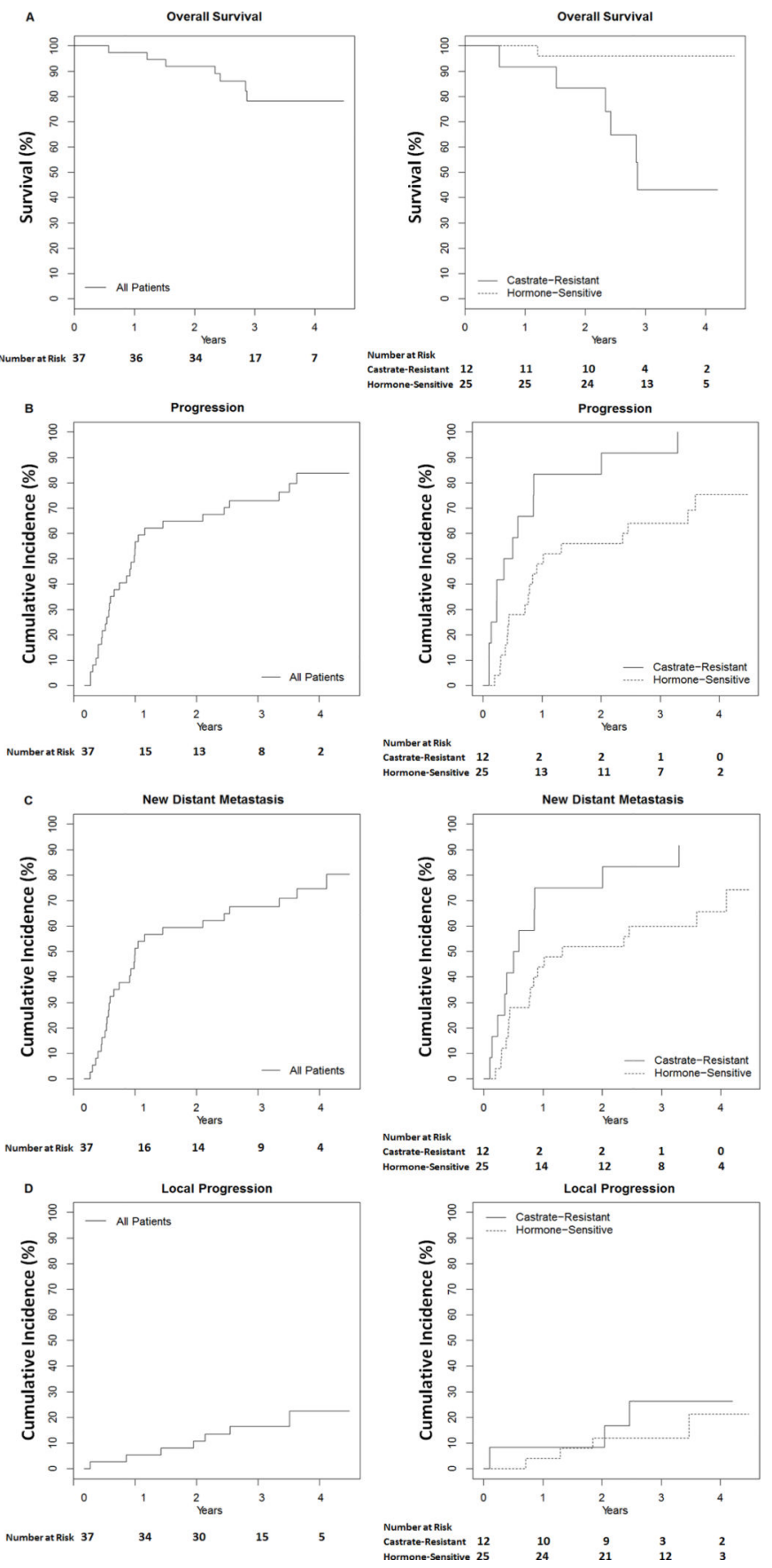

FIGURE 1. Survival and Cumulative Incidence Curves

Kaplan-Meier survival plot (A) and cumulative incidence curves with competing risk of death (B-D) showing clinical outcomes for the entire study group (left) and dichotomized by castrate-resistant status (right). 


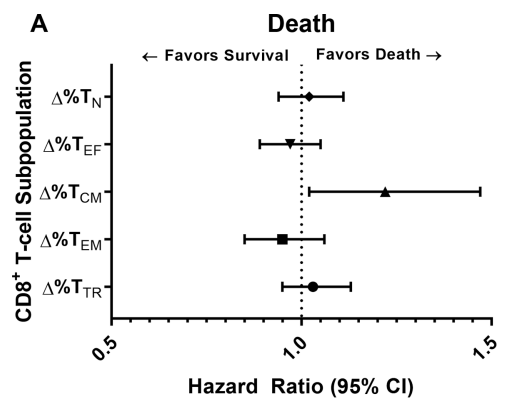

HR $(95 \% \mathrm{CI}) \quad P$ Value

$1.02(0.94-1.11) \quad 0.60$

$0.97(0.89-1.05) \quad 0.40$

$1.22(1.02-1.47) \quad 0.03$

$0.95(0.85-1.06) \quad 0.34$

$1.03(0.95-1.13) \quad 0.44$

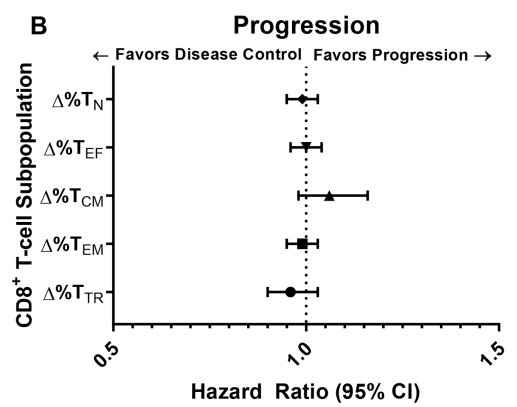

HR $(95 \%$ CI) $\quad P$ Value

$0.99(0.95-1.03) \quad 0.67$

$1.00(0.96-1.04) \quad 0.85$

$1.06(0.98-1.16) \quad 0.15$

$0.99(0.95-1.03) \quad 0.59$

$0.96(0.90-1.03) \quad 0.29$

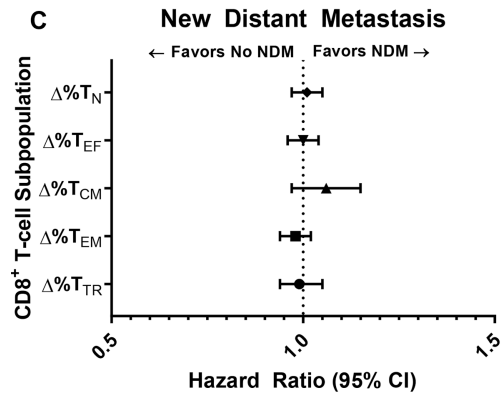

HR $(95 \% \mathrm{CI}) \quad P$ Value

$1.01(0.97-1.05) \quad 0.69$

$1.00(0.96-1.04) \quad 0.97$

$1.06(0.97-1.15) \quad 0.21$

$0.98(0.94-1.02) \quad 0.31$

$0.99(0.94-1.05) \quad 0.82$
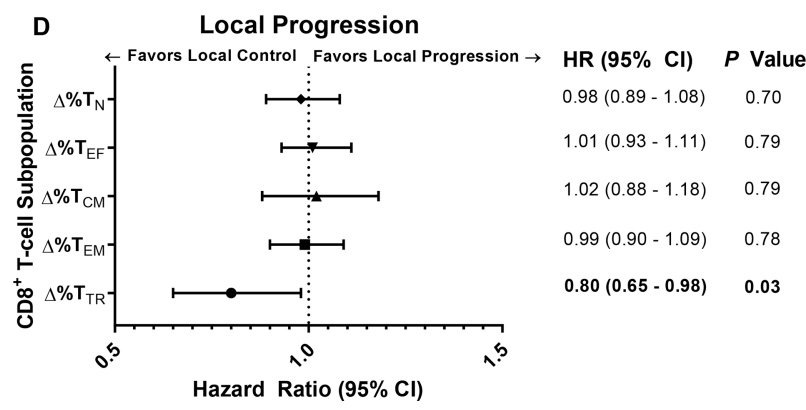

FIGURE 2. Association between the Post-mdSBRT Immunophenotypic Responses in Circulating $\mathrm{CD8}^{+}$T-cell Subpopulations and Clinical Outcomes

Forest plots demonstrating the association between changes in the relative percentage of circulating $\mathrm{CD}^{+} \mathrm{T}$-cell subpopulations and clinical outcomes including (A) death, (B) any disease progression, local or distant, (C) new distant metastasis, and (D) local progression. All plots shown convey the results of immunophenotypic changes that occurred between baseline and day 14 after metastasis-directed stereotactic body radiation therapy. Hazard ratios are per 1 unit increase in the variable. $\mathrm{T}_{\mathrm{N}}\left(\mathrm{CD} 8^{+} \mathrm{CCR} 7^{+} \mathrm{CD} 45 \mathrm{RA}^{+}\right)$, Naïve T-cell; $\mathrm{T}_{\mathrm{EF}}$ $\left(\mathrm{CD}^{+} \mathrm{CCR}^{-}{ }^{-} \mathrm{CD} 45 \mathrm{RA}^{+}\right)$, Effector T-cell; $\mathrm{T}_{\mathrm{CM}}\left(\mathrm{CD}^{+} \mathrm{CCR}^{+} \mathrm{CD} 45 \mathrm{RA}^{-}\right)$, Central Memory T- 
cell; $\mathrm{T}_{\mathrm{EM}}\left(\mathrm{CD} 8^{+} \mathrm{CCR} 7^{-} \mathrm{CD} 45 \mathrm{RA}^{-}\right)$, Effector Memory T-cell; $\mathrm{T}_{\mathrm{TR}}\left(\mathrm{CD} 8^{+} \mathrm{PD}-1^{+} \mathrm{CD} 11 \mathrm{a}^{\text {high }}\right)$, Tumor-Reactive T-cell; CI, Confidence Interval. 

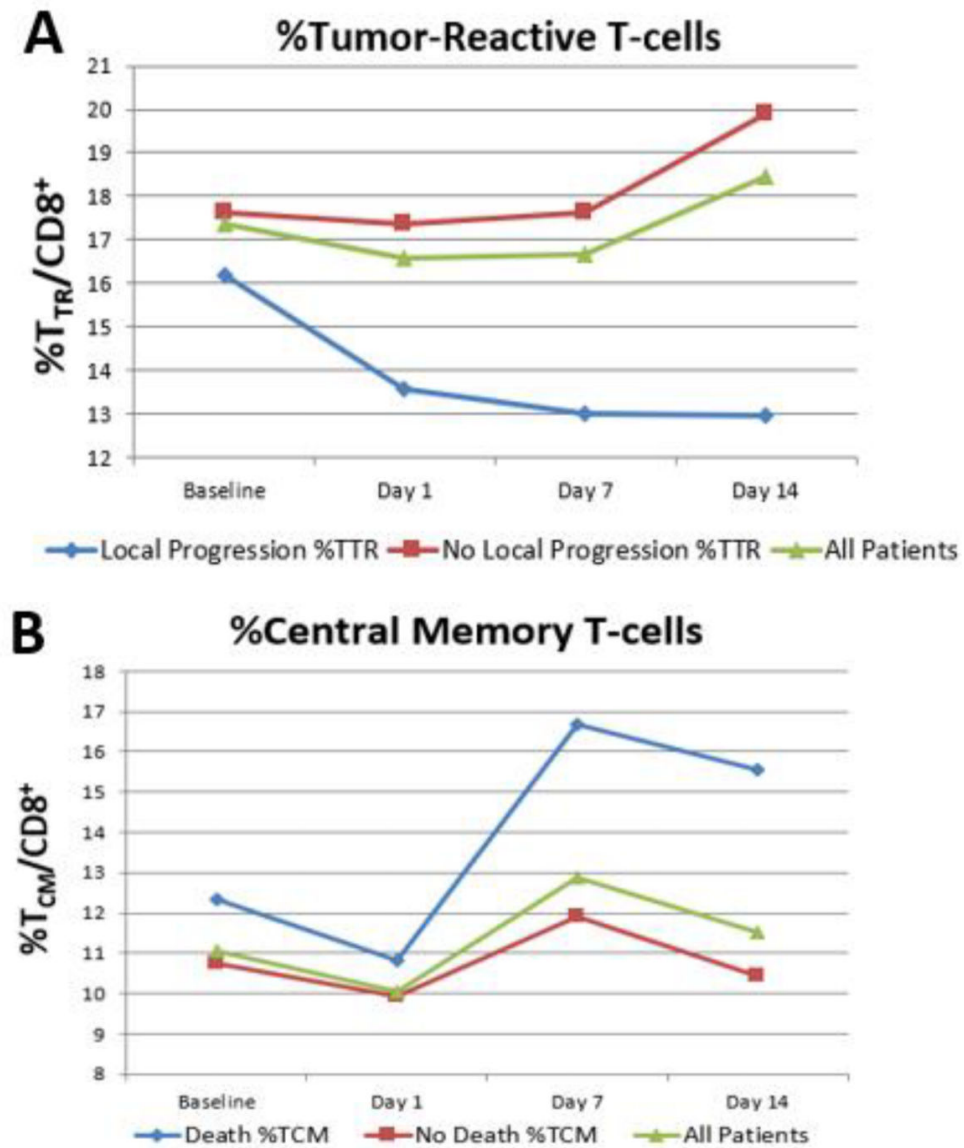

C

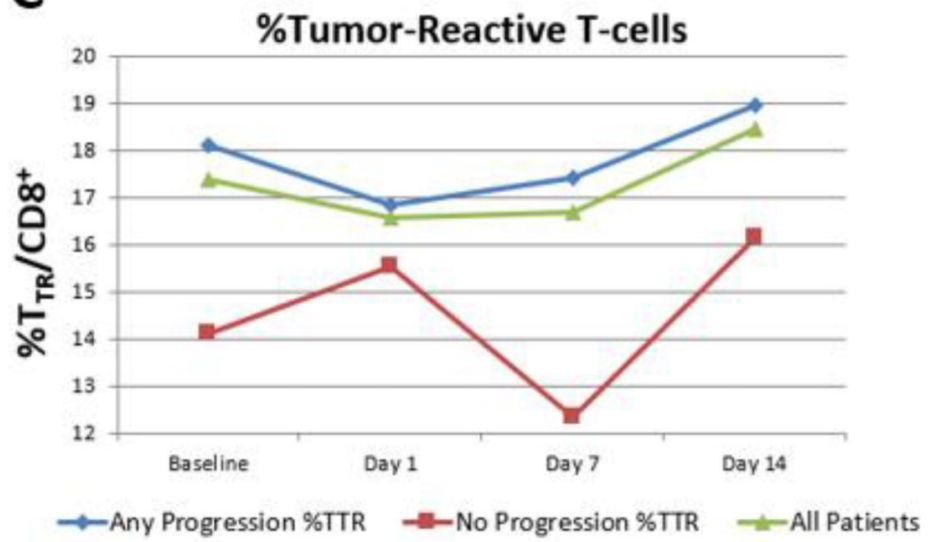

FIGURE 3. Changes in CD8+ T-cell subpopulations that correlate with significant clinical outcomes.

Graphs demonstrate the mean percentage change in (A) Tumor-Reactive $\left(\mathrm{T}_{\mathrm{TR}}\right) \mathrm{T}$-cells as a function of time at baseline before metastasis-directed SBRT and 1, 7 and 14 days after SBRT for patients that experienced local progression (blue), did not experience local progression (red), and all patients (green). (B) Mean percentage change in Central Memory $\left(\mathrm{T}_{\mathrm{CM}}\right)$ T-cells as a function of time at baseline before metastasis-directed SBRT and 1, 7, and 14 days after SBRT for patients that died (blue), remain alive (red), and all patients (green). (C) Mean percentage change in Tumor-Reactive $\left(\mathrm{T}_{\mathrm{TR}}\right) \mathrm{T}$-cells as a function of time 
at baseline before metastasis-directed SBRT and 1, 7, and 14 days after SBRT for patients that experienced any progression (blue), did not experience progression (red), and all patients (green). 
Table 1.

Patient, Tumor and Treatment Characteristics

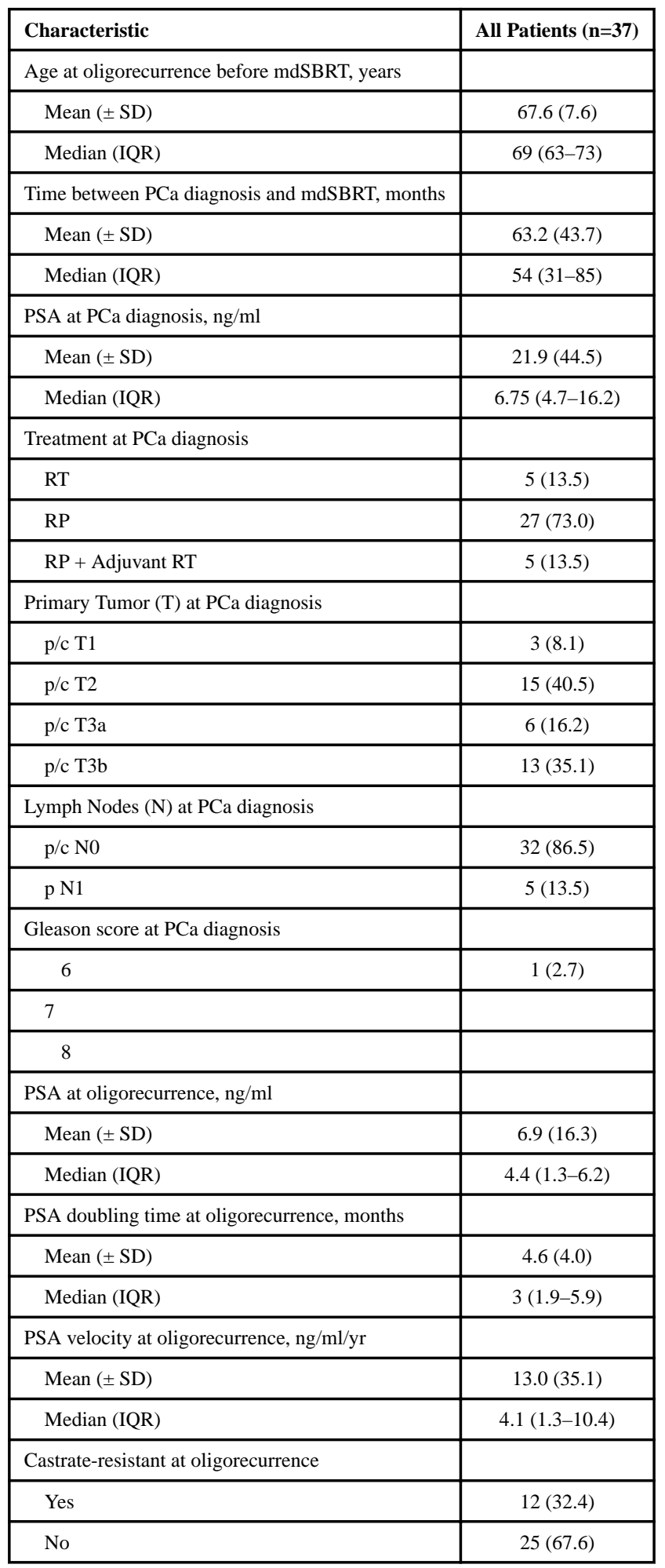




\begin{tabular}{|l|c|}
\hline Characteristic & All Patients (n=37) \\
\hline Number of mdSBRT treated site(s) & $31(83.8)$ \\
\hline 1 & $4(10.8)$ \\
\hline 2 & $2(5.4)$ \\
\hline 3 & \\
\hline Location of mdSBRT treated site(s) & $25(67.6)$ \\
\hline Non-spinal osseous & $5(13.5)$ \\
\hline Spinal & $1(2.7)$ \\
\hline Lymph node & $6(16.2)$ \\
\hline Spinal + Non-spinal osseous & \\
\hline Dose and Fractionation of mdSBRT & $11(29.7)$ \\
\hline 30 Gy in 3 Fractions & $2(5.4)$ \\
\hline 24 Gy in 1 Fraction & $20(54.1)$ \\
\hline 18 Gy in 1 Fraction & $4(10.8)$ \\
\hline 16 Gy in 1 Fraction & $19(51.4)$ \\
\hline Androgen Deprivation Therapy & $18(48.6)$ \\
\hline Concurrent with and adjuvant to mdSBRT & \\
\hline Delayed until additional progression & \\
\hline
\end{tabular}

Abbreviations: mdSBRT, metastasis-directed Stereotactic Body Radiation Therapy; PSA, prostate specific antigen; SD, Standard Deviation; IQR, Interquartile Range. 
Table 2.

Local Progression Rates by mdSBRT Dose and Fractionation

\begin{tabular}{|l|c|c|c|}
\hline Dose and Fractionation & No. of Local Progressions (n=7) & $\begin{array}{l}\text { No. of Patients per Dose Regimen } \\
(\mathbf{n = 3 7})\end{array}$ & $\begin{array}{l}\text { Local Progression Rate by Dose } \\
\text { Regimen* }\end{array}$ \\
\hline 30 Gy in 3 Fractions & $2(28.6)$ & $11(29.7)$ & $18.2 \%$ \\
\hline 24 Gy in 1 Fraction & $0(0.0)$ & $2(5.4)$ & $0.0 \%$ \\
\hline 18 Gy in 1 Fraction & $4(57.1)$ & $20(54.1)$ & $20.0 \%$ \\
\hline 16 Gy in 1 Fraction & $1(14.3)$ & $4(10.8)$ & $25.0 \%$ \\
\hline
\end{tabular}

Abbreviations: mdSBRT, metastasis-directed Stereotactic Body Radiation Therapy; No., number.

No difference between groups by Cox regression $(P=0.96)$. 
Table 3.

Percentage of $\mathrm{CD}^{+}$T-cell Subpopulations at the Four Evaluable Time Points

\begin{tabular}{|c|c|c|c|c|c|c|c|}
\hline CD8 $^{+}$T-cell Subpopulation & Baseline $(n=37)$ & $\begin{array}{c}\text { Day } 1 \text { after } \\
\text { mdSBRT } \\
(n=37)\end{array}$ & $P$ Value $*$ & $\begin{array}{c}\text { Day } 7 \text { after } \\
\text { mdSBRT } \\
(n=37)\end{array}$ & $P$ Value ${ }^{\dagger}$ & $\begin{array}{c}\text { Day } 14 \text { after } \\
\text { mdSBRT } \\
(n=37)\end{array}$ & $P$ Value ${ }^{\S}$ \\
\hline \multicolumn{8}{|l|}{$\% \mathrm{~T}_{\mathrm{N}}$} \\
\hline Mean $( \pm \mathrm{SD})$ & $12.5(11.7)$ & $12.5(13.6)$ & 0.55 & $14.4(14.0)$ & 0.07 & $14.2(15.7)$ & 0.18 \\
\hline Median (IQR) & $8.8(3.4-16.9)$ & $8.8(3.6-13.4)$ & & $11.3(3.3-17.5)$ & & $9.6(3.0-16.2)$ & \\
\hline \multicolumn{8}{|l|}{$\% \mathrm{~T}_{\mathrm{EF}}$} \\
\hline Mean $( \pm$ SD) & $32.6(18.3)$ & $33.4(18.9)$ & 0.50 & $31.1(18.5)$ & 0.08 & $27.8(17.3)$ & 0.04 \\
\hline Median (IQR) & $28.8(17.5-41.2)$ & $30.0(18.6-44.4)$ & & $24.5(17.5-41.6)$ & & $26.1(15.9-32.6)$ & \\
\hline \multicolumn{8}{|l|}{$\% \mathrm{~T}_{\mathrm{CM}}$} \\
\hline Mean $( \pm \mathrm{SD})$ & $11.1(7.6)$ & $10.1(6.5)$ & 0.16 & $12.9(8.1)$ & 0.12 & $11.5(7.0)$ & 0.99 \\
\hline Median (IQR) & $10.1(5.9-14.0)$ & $8.9(5.5-14.8)$ & & $11.4(7.3-18.6)$ & & $10.6(6.7-15.9)$ & \\
\hline \multicolumn{8}{|l|}{$\% \mathrm{~T}_{\mathrm{EM}}$} \\
\hline Mean $( \pm$ SD) & $27.7(14.3)$ & $27.5(13.0)$ & 0.55 & $25.7(11.2)$ & 0.22 & $29.9(13.8)$ & 0.58 \\
\hline Median (IQR) & $28.2(16.6-39.8)$ & $27.9(18.6-35.2)$ & & $26.4(19.0-34.1)$ & & $29.7(20.7-38.9)$ & \\
\hline \multicolumn{8}{|l|}{$\% \mathrm{~T}_{\mathrm{TR}}$} \\
\hline Mean $( \pm$ SD) & $17.4(11.4)$ & $16.6(12.8)$ & 0.56 & $16.7(12.8)$ & 0.56 & $18.5(14.2)$ & 0.51 \\
\hline Median (IQR) & $14.6(9.6-20.0)$ & $13.4(10.6-21.5)$ & & $12.2(8.3-22.0)$ & & $14.0(9.0-23.7)$ & \\
\hline
\end{tabular}

Abbreviations: mdSBRT, metastasis-directed Stereotactic Body Radiation Therapy; $\mathrm{T}_{\mathrm{N}}\left(\mathrm{CD} 8{ }^{+} \mathrm{CCR} 7^{+} \mathrm{CD} 45 \mathrm{RA}{ }^{+}\right)$, Naïve Tcell; $\mathrm{TEF}$ $\left(\mathrm{CD} 8^{+} \mathrm{CCR} 7^{-}{ }^{-} \mathrm{CD} 45 \mathrm{RA}^{+}\right)$, Effector T-cell; $\mathrm{T}_{\mathrm{CM}}\left(\mathrm{CD} 8^{+} \mathrm{CCR}^{+}{ }^{+} \mathrm{CD} 45 \mathrm{RA}^{-}\right)$, Central Memory T-cell; $\mathrm{T}_{\mathrm{EM}}\left(\mathrm{CD} 8^{+} \mathrm{CCR} 7^{-} \mathrm{CD}^{-} \mathrm{RA}^{-}\right), \mathrm{Effector}^{-}$ Memory T-cell; TTR $\left(\mathrm{CD}^{+}{ }^{+} \mathrm{PD}-1^{+} \mathrm{CD} 11 \mathrm{a}^{\text {high }}\right)$, Tumor-Reactive T-cell; SD, Standard Deviation; IQR, Interquartile Range.

${ }^{*} P$ Values based on paired sample t-test comparing means between baseline and day $1 *, 7 \dagger$, or $14 \S$ after mdSBRT, respectively.

${ }^{\dagger} P$ Values based on paired sample t-test comparing means between baseline and day $1^{*}, 7 \dagger$, or $14 \S$ after mdSBRT, respectively.

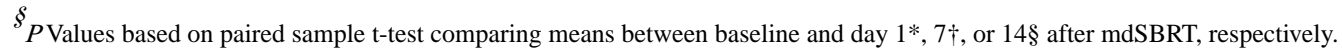

